07

\title{
Лавинный пробой в 4H-SiC диодах Шоттки: вопросы надежности
}

\author{
(С П.А. Иванов, А.С. Потапов, Н.М. Лебедева, И.В. Грехов \\ Физико-технический институт им. А.Ф. Иофрфе РАН, \\ 194021 Санкт-Петербург, Россия \\ e-mail: Pavel.Ivanov@mail.ioffe.ru
}

Поступило в Редакцию 4 марта 2020 г.

В окончательной редакции 11 марта 2020 г.

Принято к публикации 12 марта 2020 г.

\begin{abstract}
Рассмотрены вопросы, связанные с надежностью силовых $4 H-\mathrm{SiC}$ диодов Шоттки при кратковременных электрических перегрузках в обратном направлении (при работе диодов в импульсном лавинном режиме). В частности, обсуждается влияние неоднородности лавинного пробоя по площади диода на величину максимальной лавинной энергии (МЛЭ), которая может быть рассеяна диодом до того, как в нем произойдет вторичный тепловой пробой. Для оценки того, насколько однороден лавинный пробой, предложено сравнивать измеренную импульсную обратную вольт-амперную характеристику (BAX) диода с расчетной ВАХ идеального квазиодномерного диода.

Проведены измерения обратных $\mathrm{BAX}$ промышленных 4H-SiC ДШ: через диоды пропускались одиночные импульсы лавинного тока длительностью $\sim 1 \mu \mathrm{s}$; в процессе измерений амплитуда импульсов поднималась до величин, при которых происходил катастрофический отказ диодов. Показано, что увеличенное дифференциальное сопротивление диодов на лавинном участке ВАХ и пониженное экстраполированное напряжение пробоя (по сравнению с их расчетными значениями для идеальных диодов) предсказывают снижение величины МЛЭ.
\end{abstract}

Ключевые слова: карбид кремния, диод Шоттки, лавинный пробой, надежность.

DOI: $10.21883 / J T F .2020 .12 .50132 .71-20$

\section{Введение}

В настоящее время в мире промышленно производятся силовые высоковольтные диоды Шоттки (ДШ) на основе карбида кремния $(4 H-\mathrm{SiC})$. Выпускаемые $4 H$-SiC ДШ рассчитаны на обратное напряжение от 600 до $1700 \mathrm{~V}$ и прямой ток от одного до нескольких десятков ампер (см., например, [1]). В силовой электронике $4 H$-SiC ДШ активно приходят на замену кремниевым быстровосстанавливающимся диодам (БВД). (При сравнимом с $\mathrm{Si}$ БВД обратном напряжении $4 \mathrm{H}-\mathrm{SiC}$ ДШ имеют намного меньшие статические и динамические потери.) В этой связи много внимания стало уделяться надежности 4H-SiC ДШ, в частности, способности выдерживать без отказа кратковременные электрические перегрузки.

Очень часто отказы диодов происходят при смещении в обратном направлении; отказы могут провоцироваться внезапным перенапряжением и развитием сначала лавинного, а затем вторичного теплового пробоя. Количественно устойчивость диодов к электротепловому пробою принято характеризовать максимальной лавинной энергией (МЛЭ), которая может быть рассеяна диодом до того, как происходит его катастрофический отказ (величину МЛЭ определяют, как правило, при пропускании через диод одиночного импульса лавинного тока). Некоторые производители силовых 4H-SiC ДШ уже сертифицируют свою продукцию по величине МЛЭ (см., например, [2]).
Отказы 4H-SiC ДШ, обусловленные пробоем в обратном направлении, в первую очередь связаны с эффективностью работы охранного контура, который применяется для подавления преждевременного краевого пробоя. Для планарных 4H-SiC ДШ были разработаны разные конструктивно-технологические решения охранного контура, позволяющие повысить напряжение пробоя вплоть до теоретически предельной величины (см., например, [3]). В последнее время появилось много работ, посвященных измерениям МЛЭ в $4 H$-SiC ДШ разной конструкции [4-7]. Наилучшие из известных нам результатов достигнуты в работе [7], в которой эффективность охранного контура была близкой к 100\%, а лавинный пробой был однородным по всей площади. Для $650-\mathrm{V}$ диодов измеренная МЛЭ составила $5 \mathrm{~J} / \mathrm{cm}^{2}$ при длительности импульса лавинного тока $4 \mu \mathrm{s}$ и $20 \mathrm{~J} / \mathrm{cm}^{2}$ при длительности импульса $400 \mu \mathrm{s}$ (помимо однородности пробоя, на саморазогрев диода оказывают влияние длительность импульса и условия теплоотвода). Однородность пробоя была зафиксирована путем визуального наблюдения предпробойной электролюминесценции (наблюдение проводилось со стороны подложки; для этого в катодном контакте специально оставлялись окна для вывода света). Вообще говоря, делать выводы об однородности пробоя в готовых (корпусированных) диодах можно только на основе данных электрических измерений. В настоящей работе продемонстрирован такой подход, основанный на измерениях обратных вольтамперных характеристик (BAX) в режиме пробоя на коротких импульсах. 


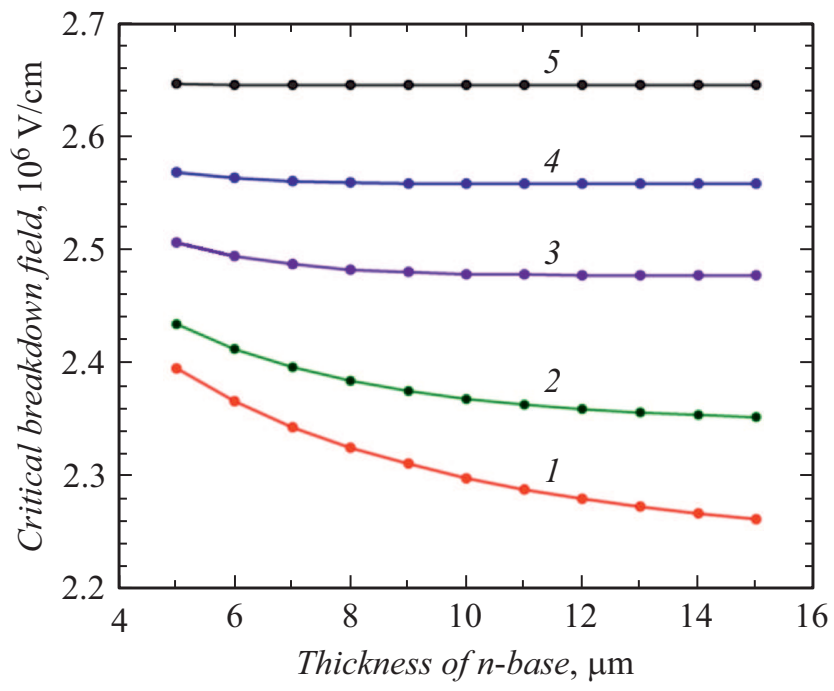

Pис. 1. Зависимость критической напряженности поля от толщины $n$-базы для различных концентраций доноров: $1-10^{15}$, $2-2 \cdot 10^{15}, 3-4 \cdot 10^{15}, 4-6 \cdot 10^{15}, 5-9 \cdot 10^{15} \mathrm{~cm}^{-3}$.

\section{1. Критическая напряженность поля лавинного пробоя в ДШ С „проколом“ $n$-базы}

ДШ на основе 4H-SiC обычно конструируют так, чтобы область пространственного заряда (ОПЗ) полностью перекрывала блокирующую $n$-базу $n-n^{+}$-структуры при напряжении, составляющем около половины от пробивного (это так называемый диод с „проколом“ $n$-базы). В диоде с „проколом“ базы критическое поле пробоя $E_{B}$ должно зависеть от уровня легирования и толщины $n$-базы и может быть определено из условия лавинного пробоя

$$
\int_{0}^{d} \alpha_{n} \exp \left[-\int_{x}^{d}\left(\alpha_{n}-\alpha_{p}\right) d x^{\prime}\right] d x=1,
$$

где $d$ - толщина блокирующей $n$-базы, $\alpha_{n}$ и $\alpha_{p}-$ коэффициенты ионизации электронов и дырок соответственно. В $4 H-\mathrm{SiC}$ коэффициенты ионизации хорошо описываются по модели [8]:

$$
\begin{aligned}
& \alpha_{n}=\alpha_{n o} \exp \left(-E_{n} / E\right), \\
& \alpha_{p}=\alpha_{p o} \exp \left(-E_{p} / E\right),
\end{aligned}
$$

со следующими параметрами [9]: $\alpha_{\text {no }}=1.76 \cdot 10^{8} \mathrm{~cm}^{-1}$, $\alpha_{p o}=3.41 \cdot 10^{8} \mathrm{~cm}^{-1}, E_{n}=3.3 \cdot 10^{7} \mathrm{~V} / \mathrm{cm}, E_{p}=2.5 \cdot 10^{7} \mathrm{~V} / \mathrm{cm}$.

При пробое распределение поля $E$ в $n$-базе записывается в следующем виде:

$$
E=E_{B}-q N x / \varepsilon,
$$

где $q-$ элементарный заряд, $\varepsilon-$ диэлектрическая проницаемость полупроводника, $N$ - концентрация доноров в $n$-базе. Подставляя (2), (3) и (4) в (1), можно получить следующее интегральное уравнение относительно $E_{B}$ (оно решалось средствами Mathcad):

$$
\begin{aligned}
& \int_{0}^{d} \alpha_{n 0} \exp \left(-\frac{E_{n}}{E_{B}-q N x / \varepsilon}\right) \\
& \quad \times \exp \left(-\int_{x}^{d}\left[\alpha_{n o} \exp \left(-\frac{E_{n}}{E_{B}-q N x^{\prime} / \varepsilon}\right)\right.\right. \\
& \left.\left.-\alpha_{p o} \exp \left(-\frac{E_{p}}{E_{B}-q N x^{\prime} / \varepsilon}\right)\right] d x^{\prime}\right) d x=1 .
\end{aligned}
$$

Как видно, в уравнение (5) входят два параметра блокирующей $n$-базы - концентрация доноров $N$ и толщина $d$. На рис. 1 показаны рассчитанные зависимости $E_{B}(N, d)$.

\section{2. Идеализированная BAX обратносмещенного ДШ}

На рис. 2 показана идеализированная ВАХ обратносмещенного диода. Важнейшими электрическими параметрами являются напряжение лавинного пробоя $\left(V_{B}\right)$ и дифференциальное сопротивление на лавинном участке $\mathrm{BAX}$ - лавинное сопротивление $\left(R_{\text {aval }}\right)$. На показанной ВАХ при $V<V_{B}$ ток $I=0$, а при $V>V_{B}$ ток растет при увеличении напряжения так, что $\Delta I=\Delta V / R_{\text {aval }}$. Соответствующие распределения электрического поля $E$ в ОПЗ квазиодномерной $n-n^{+}$-структуры показаны на рис. 3 для двух случаев: 1$) V=V_{B}$ и 2) $V>V_{B}$ (лавинное умножение происходит в очень узком слое, там, где электрическое поле достигает критического значения $\left.E_{B}[10]\right)$. При известной величине $E_{B}$ можно легко рассчитать напряжение пробоя и лавинное сопротивление. При $V=V_{B}$ (начало пробоя), когда градиент поля в ОПЗ задается только концентрацией положительно заряженных ионизированных доноров $N$ (концентрация свободных электронов в ОПЗ еще мала: $n \ll N)$, напря-

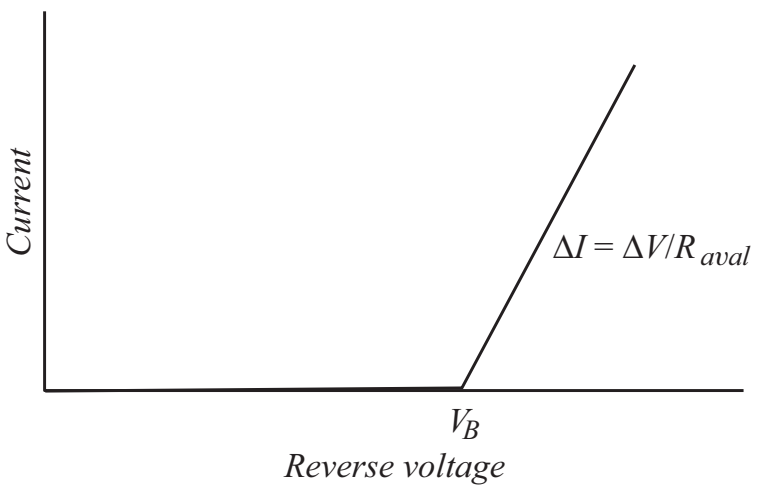

Рис. 2. Идеализированная ВАХ обратносмещенного ДШ. 
Конструктивные данные и предельные (расчетные) электрические параметры диодов CSD02060A и C3D02060A

\begin{tabular}{l|c|c}
\hline \multicolumn{1}{|c|}{ Параметры } & CSD02060A & C3D02060A \\
\hline Концентрация доноров в $n$-базе $n, \mathrm{~cm}^{-} 3$ & $5.2 \cdot 10^{15}$ & $10^{16}$ \\
Толщина $n$-базы $d, \mu \mathrm{m}$ & 6.1 & 5 \\
Площадь анода $S, \mathrm{~cm}^{2}$ & $10^{-2}$ & $4.7 \cdot 10^{-3}$ \\
Напряженность поля пробоя $E_{B}, \mathrm{~V} / \mathrm{cm}$ & $2.5 \cdot 10^{6}$ & $2.65 \cdot 10^{6}$ \\
Напряжение пробоя $V_{B}, \mathrm{~V}$ & 1355 & 1180 \\
Лавинное сопротивление $R_{\text {aval }}, \Omega$ & 2.5 & 3.6
\end{tabular}

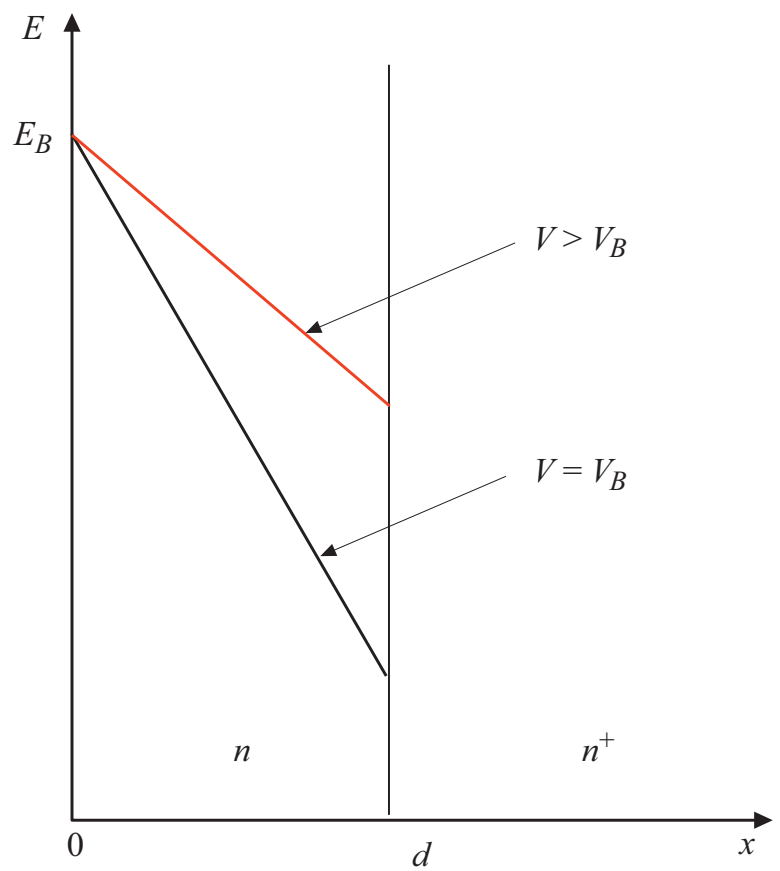

Рис. 3. Распределения электрического поля в ОПЗ обратносмещенного ДШ на основе квазиодномерной полупроводниковой $n-n^{+}$-структуры с ,проколом“ $n$-базы.

жение пробоя рассчитывается как

$$
V_{B}=E_{B} d-q N d^{2} / 2 \varepsilon \text {. }
$$

При $V>V_{B}$, когда концентрация свободных электронов в ОПЗ становится существенной $(n>0.1 N)$, в уравнении Пуассона для ОПЗ необходимо учитывать отрицательный объемный заряд свободных электронов. (Отметим, что при полях $E>10^{6} \mathrm{~V} / \mathrm{cm}$ электроны в $4 H-\mathrm{SiC}$ дрейфуют в ОПЗ с постоянной насыщенной скоростью $v=8 \cdot 10^{6} \mathrm{~cm} / \mathrm{s}[11,12]$, и их концентрация n в пределах ОПЗ не зависит от координаты.) В присутствии свободных электронов градиент поля в ОПЗ уменьшается, так что падение напряжения возрастает:

$$
V=E_{B} d-q(N-n) d^{2} / 2 \varepsilon .
$$

Лавинный ток $I$ и концентрация электронов $\mathrm{n}$ связаны простой зависимостью:

$$
I=q n v S
$$

где $S$ - площадь анода. Из формул (6)-(8) можно вывести следующее выражение для лавинного сопротивления диода:

$$
R_{\text {aval }}=\left(V-V_{B}\right) / I=d^{2} / 2 \varepsilon v S \text {. }
$$

Таким образом, в ДШ с „проколом“ $n$-базы лавинное сопротивление не зависит от уровня легирования и задается только ее толщиной $(d)$ и площадью $(S)$.

До сих пор лавинный пробой рассматривался как однородный (лавинный ток $I$ однородно распределен по всей площади диода). Однако в случае недостаточно эффективного охранного контура пробой остается краевым. В этом случае измеренное напряжение пробоя $\left(V_{B}^{\prime}\right)$ будет меньше, а измеренное дифференциальное сопротивление $\left(R_{\text {aval }}^{\prime}\right)$ будет больше значений, рассчитанных по формулам (6) и (9) соответственно. То есть, измеряя $V_{B}^{\prime}$ и $R_{\text {aval }}^{\prime}$ и сравнивая их с расчетными значениями, можно делать прогнозы относительно устойчивости диодов к электротепловому пробою.

\section{4H-SiC ДШ, использованные для исследований}

Экспериментальные исследования проводились с использованием промышленных диодов CSD02060A и C3D02060A $(600 \mathrm{~V}, 2 \mathrm{~A})$, производимых в разное время компанией CREE/WOLFSPEED [13,14] (диоды CSD02060A производились еще в 2000-х годах, а диоды C3D02060A выпускаются в настоящее время). Размеры чипов и площадь анода определялись после вскрытия корпуса ТО-220, а концентрация доноров в блокирующей $n$-базе $(N)$ и ее толщина $(d)$ - по измеренным вольт-фарадным характеристикам (ВФХ). По полученным данным рассчитывались критическая напряженность поля $E_{B}$, напряжение пробоя $V_{B}$ и лавинное сопротивление $R_{\text {aval }}$. Перечисленные конструктивные данные и предельные (расчетные) электрические параметры диодов сведены в таблицу. Как видно, конструктивные данные диодов существенно отличаются (хотя диоды имеют одинаковые номинальные значения напряжения и тока). В более современных диодах уровень легирования выше, а толщина базы и площадь чипа меньше. Это заранее говорит о том, что в современном варианте охранная система должна быть более эффективной. 


\section{4. Измерения ВАХ и МЛЭ в режиме пробоя на коротких импульсах}

Импульсные ВАХ диодов в обратном направлении и МЛЭ измерялись с помощью схемы, показанной на рис. 4. В зарубежной литературе данная схема получила название Unclamped Inductive Switching (UIS). Источником импульсного питания в схеме служит конденсатор $C$ большой емкости, заряженный до напряжения $V_{0}$ (величина $V_{0}$ выбирается намного меньше, чем статическое напряжение пробоя диода $\left.V_{B}\right)$. Исходно к исследуемому диоду приложено обратное напряжение $V_{0}$; это же напряжение приложено к стоку закрытого IGBT-транзистора (T). Когда транзистор T открывается импульсом напряжения $V_{G}$ на затворе, конденсатор $C$ разряжается через открытый транзистор, индуктивность $L$ и сопротивление $R_{2}$. Пиковый зарядный ток в индуктивности $L$ может варьироваться изменением длительности открытого состояния транзистора $\mathrm{T}$ (изменением длительности импульса $V_{G}$ ). После закрывания транзистора $\mathrm{T}$ ток из него быстро перебрасывается в исследуемый диод (DUT) вместе с последовательно включенным измерительным резистором $R_{3}$. При этом на диоде генерируется обратное напряжение, превышающее напряжение статического пробоя $V_{B}$. Далее по мере разряда индуктивности $L$ ток падает, а напряжение остается выше $V_{B}$. И наконец, когда ток уменьшается практически до нуля, напряжение также снижается до исходной величины $V_{0}$. Временные диаграммы напряжения на диоде и тока через него записывались двухканальным

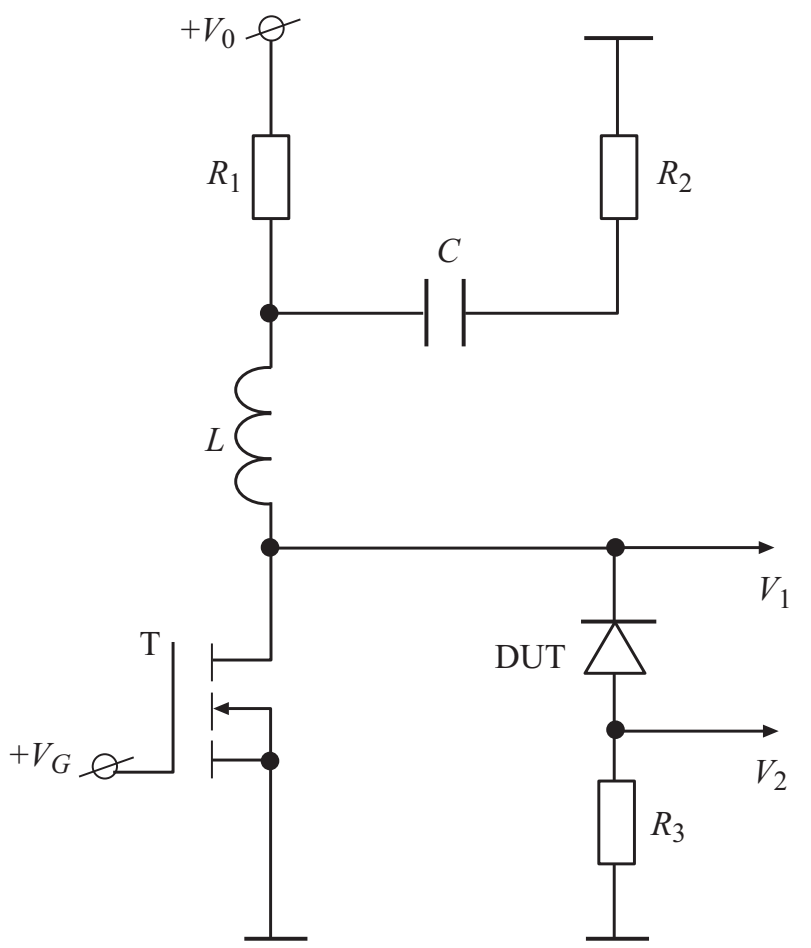

Pис. 4. Схема UIS-измерений импульсных обратных BAX $4 H$-SiC ДШ в режиме пробоя.

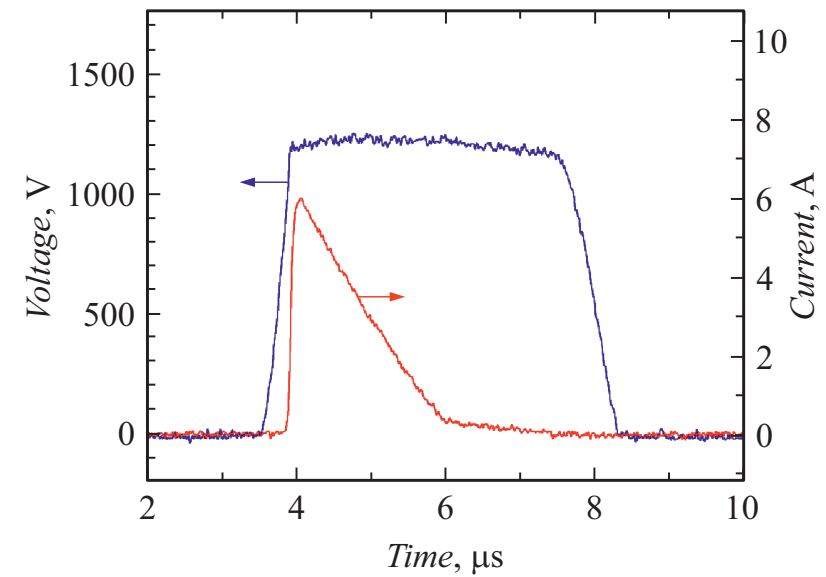

Рис. 5. Осциллограммы напряжения и тока, измеренные в диоде C3D02060A в режиме обратимого лавинного пробоя.

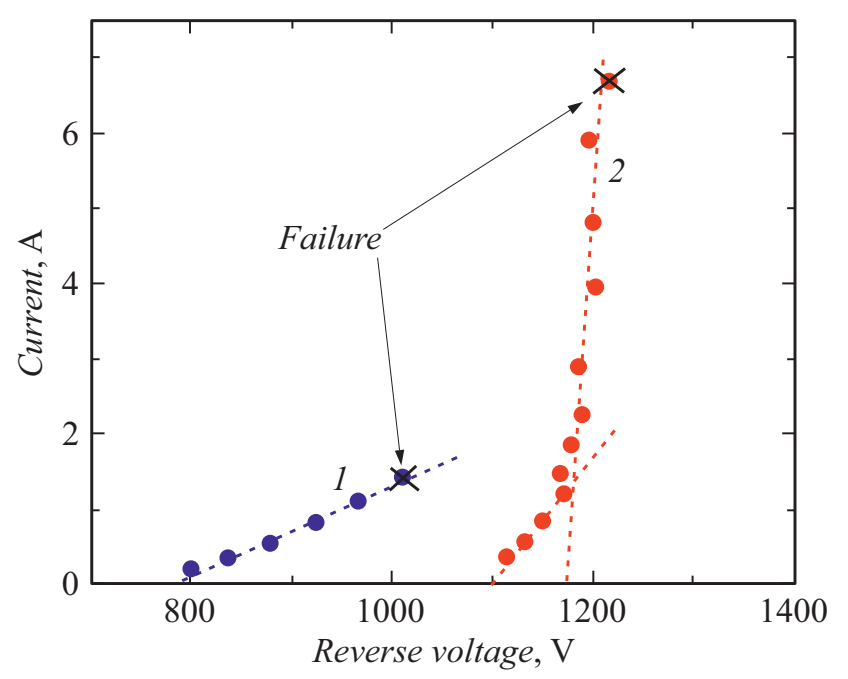

Рис. 6. Обратные ВАХ диодов CSD02060A (1) и C3D02060A (2).

цифровым осциллографом. Мгновенную мощность $P(t)$, которая рассеивается во время лавинного процесса, можно рассчитать путем перемножения мгновенных величин тока $I(t)$ и напряжения $V(t)$. Полную энергию, рассеянную при прохождении импульса лавинного тока, можно вычислить как интеграл от мощности по времени.

На рис. 5 для примера показаны осциллограммы напряжения и тока, измеренные в диоде C3D02060A в режиме обратимого лавинного пробоя. ВАХ диодов CSD02060A и C3D02060A (рис. 6) строились по пиковым значениям напряжения и тока.

В диоде CSD02060A ток в пределах от 0.3 до $1.4 \mathrm{~A}$ линейно растет с ростом напряжения. Дифференциальное сопротивление на этом участке составляет $170 \Omega$ и намного (в 68 раз) превышает расчетное значение $(2.5 \Omega)$. Экстраполяция линейной зависимости к $I=0$ дает напряжение пробоя $790 \mathrm{~V}$, что составляет только $58 \%$ от расчетного значения $(1355 \mathrm{~V})$. Получен- 


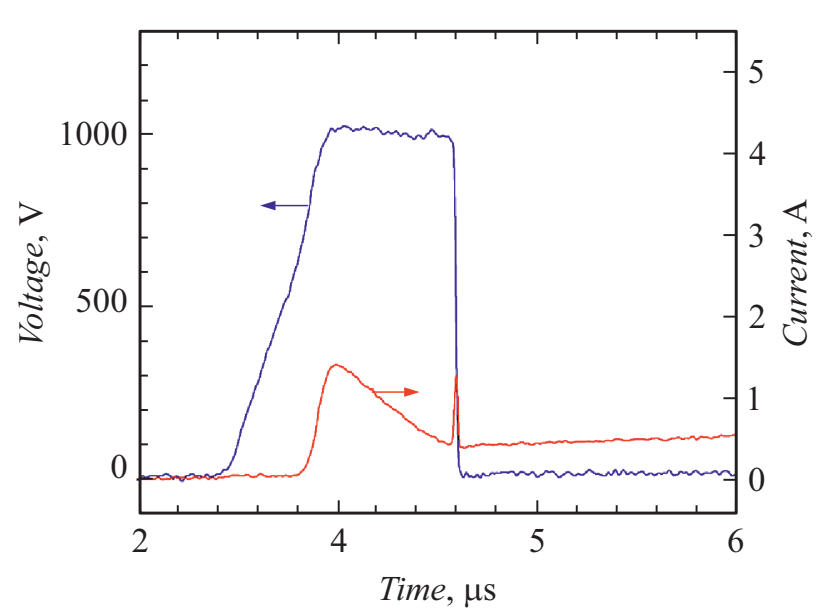

Рис. 7. Осциллограммы напряжения и тока, демонстрирующие развитие электротеплового пробоя в диоде CSD02060A.

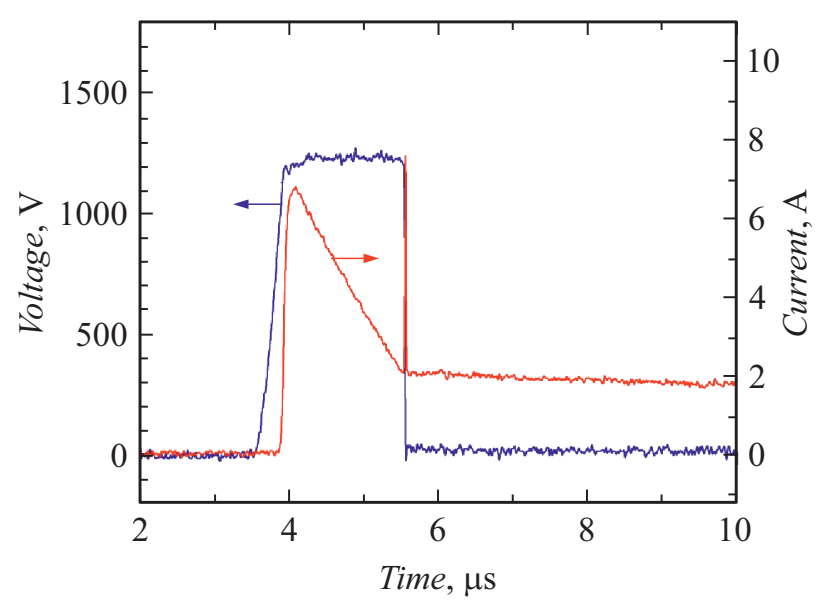

Рис. 8. Осциллограммы напряжения и тока, демонстрирующие развитие электротеплового пробоя в диоде C3D02060A.

ные результаты свидетельствуют о том, что в диодах CSD02060 пробой имеет сугубо неоднородный характер. Скорее всего, он происходит в периферийной области, и это связано с недостаточно эффективной охранной системой. Из-за сильной локализации лавинного тока можно было ожидать развития преждевременного электротеплового пробоя. Действительно, при амплитуде тока 1.4 А произошел катастрофический отказ диода. На рис. 7 показаны осциллограммы, демонстрирующие развитие в диоде электротеплового пробоя. Как видно, в некоторый момент времени, когда ток спадает уже на две трети от своего амплитудного значения, наблюдается резкий бросок тока и такой же резкий спад напряжения. МЛЭ, рассчитанная из зависимостей $I(t)$ и $V(t)$ до момента времени, при котором произошел отказ, составляет около $1.1 \mathrm{~mJ}\left(0.11 \mathrm{~J} / \mathrm{cm}^{2}\right)$.

В диоде C3D02060A на начальном участке BAX (от 0.3 до $1.5 \mathrm{~A}$ ) ток линейно растет с ростом напряжения. Дифференциальное сопротивление на этом участке составляет $70 \Omega$. Экстраполированное напряжение про- боя $(1100 \mathrm{~V})$ составляет уже $93 \%$ от расчетного значения $(1180 \mathrm{~V})$. В пределах от 2 до 7 А ток круто растет с ростом напряжения. Дифференциальное сопротивление на этом участке $(5 \Omega)$ довольно близко к расчетному значению $(3.6 \Omega)$, а экстраполированное напряжение пробоя $(1175 \mathrm{~V})$ составляет почти $100 \%$ от расчетного значения $(1180 \mathrm{~V})$. Очевидно, что на крутом участке BAX лавинный пробой однороден по площади. Поэтому от диодов C3D02060A можно было ожидать большей, по сравнению с диодами CSD02060, устойчивости к отказам вследствие электротеплового пробоя. Действительно, катастрофический отказ диода наступил при амплитуде тока 6.8 А (рис. 8). МЛЭ, рассчитанная из зависимостей $I(t)$ и $V(t)$ до момента времени, при котором произошел отказ, составляет около $5.8 \mathrm{~mJ}$. Удельная МЛЭ составляет $1.2 \mathrm{~J} / \mathrm{cm}^{2}$, что сравнимо с данными из работы [7], если принять во внимание то, что в нашем случае длительность импульса в 3 раза меньше.

\section{Заключение}

Таким образом, контроль лавинного сопротивления 4H-SiC ДШ позволяет прогнозировать надежность диодов в плане их устойчивости к отказам вследствие вторичного теплового пробоя. При анализе надежности $4 H$-SiC ДШ важно выяснять физические причины, по которым происходят отказы. Некоторое представление о механизме отказа вследствие электротеплового пробоя можно получить, определяя локальную температуру, до которой разогревается структура диода при отказе. (Для диодов Шоттки опасным является перегрев до такой температуры, при которой подвергается деструкции барьерный металлический контакт, например, в результате плавления металла.) Во время импульсных испытаний диодов прямое измерение локальной температуры крайне затруднительно (если вообще возможно), поэтому довольно часто прибегают к численному моделированию нестационарного теплового процесса в диодах. (Оценочные тепловые расчеты проводились нами, в частности, при исследованиях диодов с $p-n$ переходом [15]; было показано, что отказ происходит при саморазогреве до такой высокой температуры, при которой концентрация собственных носителей в $4 H-\mathrm{SiC}$ сравнивается с концентрацией примесей в блокирующей базе.)

Для проведения модельных экспериментов необходимо иметь полные данные о конструкции диодных чипов. В первую очередь это относится к охранному контуру, эффективность работы которого напрямую влияет на локализацию лавинного пробоя. Следует отметить, что все производимые в мире высоковольтные $4 H$-SiC диоды Шоттки на самом деле имеют интегрированную Шоттки$(p-n)$-структуру (JBS, Junction Barrier Schottky): барьеры Шоттки в ней перемежаются с локальными $p-n$-переходами, формируемыми с помощью ионной имплантации акцепторов (см., например, [3]). В обратном 
направлении ОПЗ от соседних $p-n$-переходов смыкаются, так что система $p$-колец действует как экранирующая сетка. Благодаря эффекту экранирования область максимального электрического поля (и соответственно область лавинного умножения носителей) находится не в плоскости металлического контакта, а немного отодвинута от поверхности в объем. Это также может существенным образом влиять на пространственное распределение температуры в диоде во время нестационарного теплового процесса. В работе проведение моделирования не предполагалось по причине отсутствия детальных сведений о конструкции охранного контура и JBS-структуры в промышленных 4H-SiC ДШ.

\section{Финансирование работы}

Работа выполнена при финансовой поддержке Министерства науки и высшего образования РФ в рамках комплексного проекта по созданию высокотехнологичного производства „Создание высокотехнологичного производства кремниевых и карбидокремниевых изделий микроэлектронной техники в малогабаритных металлополимерных корпусных исполнениях типа SOT, SO и QFN“ (соглашение от 29 ноября 2019 г. № 075-11-2019-035) в организации соисполнителя НИОКТР ФТИ им. А.Ф. Иоффе.

\section{Конфликт интересов}

Авторы заявляют, что у них нет конфликта интересов.

\section{Список литературы}

[1] Электронный pecypc. Режим доступа: www.wolfspeed.com/ power/products/sic-schottky-diodes

[2] Электронный ресурс. Режим доступа: www.onsemi.com/ pub/Collateral/FFSD10120A-D.pdf

[3] T. Kimoto, J.A. Cooper. Fundamentals of silicon carbide technology: growth, characterization, devices, and applications (Wiley-IEEE Press., 2014) 538 p. DOI: $10.1002 / 9781118313534$

[4] T. Nakamura, M. Aketa, Y. Nakano, M. Sasagawa, T. Otsuka. Proc. of 2012 IEEE Energytech. (Cleveland, Ohio, 2012) p. 6.

[5] T. Basler, R. Rupp, R. Gerlach, B. Zippelius, M. Draghici. PCIM Europe 2016, international exhibition and conference for power electronics, intelligent motion, renewable energy and energy management (Nuremberg, Germany, 2016), p. 181.

[6] A. Gendron-Hansen, D. Sdrull, B. Odekirk, A.S. Kashyap, L. Starr. Mater. Sci. Forum., 924, 585 (2017).

[7] A. Konstantinov, H. Pham, B. Lee, K.S. Park, B. Kang, F. Allerstam, T. Neyer. Solid State Electron., 148, 51 (2018). https://doi.org/10.1016/j.sse.2018.07.011

[8] S. Selberherr. Analysis and simulation of semiconductor devices (Springer Verlag, Wien, NY., 1984). 296 p. DOI 10.1007/978-3-7091-8752-4
[9] T. Hatakeyama, T. Watanabe, T. Shinohe et al. Appl. Phys. Lett., 85 (8). P. 1380 (2004). https://doi.org/10.1063/1.1784520

[10] M. Levinshtein, J. Kostamovaara, S. Vainshtein. Selected Topics in Electronics and Systems, 36, 224 (2005). https://doi.org/10.1142/5877

[11] K.V. Vassilevski, K. Zekentes, A.V. Zorenko, L.P. Romanov. IEEE Electron Device Lett., 21, 485 (2000). DOI: $10.1109 / 55.870609$

[12] П.А. Иванов, А.С. Потапов, Т.П. Самсонова, И.В. Грехов. ФТП, 51 (3), 390 (2017). DOI: $10.21883 /$ FTP.2017.03.44214.8385

[13] Электронный ресурс. Режим доступа: media.digikey.com/ pdf/Data Sheets/CREE Power/CSD02060.pdf

[14] Электронный ресурс. Режим доступа: www.wolfspeed.com/ media/downloads/55/C3D02060A.pdf

[15] П.А. Иванов, Т.П. Самсонова, А.С. Потапов. ФТП, 52 (12), 1527 (2018). DOI: 10.21883/FTP.2018.12.46769.8903 